\title{
Dark mode plasmonic optical microcavity biochemical
} sensor

\author{
Cheng LI ${ }^{1}{ }^{1}$ LeI CHEN, ${ }^{1,2}$ EUAN MCLEOD, ${ }^{1}$ AND JUdiTH SU ${ }^{1,3,}{ }^{*}$ \\ ${ }^{1}$ College of Optical Sciences, University of Arizona, Tucson, AZ 85721, USA \\ ${ }^{2}$ State Key Laboratory of Information Photonics and Optical Communications, Beijing University of Posts and Telecommunication, Beijing \\ 100876, China \\ ${ }^{3}$ Department of Biomedical Engineering, University of Arizona, Tucson, AZ 85721, USA \\ *judy@optics.arizona.edu
}

\begin{abstract}
Whispering gallery mode (WGM) microtoroid optical resonators have been effectively used to sense low concentrations of biomolecules down to the single molecule limit. Optical WGM biochemical sensors such as the microtoroid operate by tracking changes in resonant frequency as particles enter the evanescent nearfield of the resonator. Previously, gold nanoparticles have been coupled to WGM resonators to increase the magnitude of resonance shifts via plasmonic enhancement of the electric field. However, this approach results in increased scattering from the WGM, which degrades its quality factor (Q), making it less sensitive to extremely small frequency shifts caused by small molecules or protein conformational changes. Here we show using simulation that precisely-positioned trimer gold nanostructures generate dark modes that suppress radiation loss and can achieve high $\left(>10^{6}\right) Q$ with an electric field intensity enhancement of 4300 , which far exceeds that of a single rod ( $\sim 2500$ times). Through an overall evaluation of a combined enhancement factor, which includes the $Q$ factor of the system, the sensitivity of the trimer system was improved $105 \times$ versus $84 \times$ for a single rod. Further simulations demonstrate that unlike a single rod system, the trimer is robust to orientation changes and has increased capture area. We also conduct stability tests to show that small positioning errors do not greatly impact the result. (c) 2018 Optical Society of America under the terms of the OSA Open Access Publishing Agreement
\end{abstract}

\section{INTRODUCTION}

Whispering gallery mode (WGM) microtoroids (Fig. 1(a)) are excellent sensors due to their ultra-high quality $(\mathrm{Q})$ factors and their ability to be integrated on chip [1-9]. These sensors when combined with frequency-locking, balanced detection, and data processing techniques are able to detect wavelength shifts $(<\sim 0.005 \mathrm{fm})$ which correspond to the detection of a biomolecule of radius $2 \mathrm{~nm}$ binding to the microtoroid $[1,2,4]$. Coupling plasmonic particles to WGM cavities is one way to improve the sensitivity of the systems [10]. These particles increase the frequency shift upon molecular binding by providing enhanced interaction between the optical field and the analyte due to localized surface plasmon resonance (LSPR) [10,11]. The scattering and absorption losses of the metal, however, cause degradation of the quality (Q)-factor of the resonance, that is, the broadening of the linewidth. Dark mode plasmonic resonances can help solve this problem [12-16], but simulation is necessary to understand the specific impact of a given plasmonic structure on the system.
Currently, due to limitations in computing performance and time consumption, a wedge-shaped model with perfect electric conductor boundary conditions is widely used in plasmonically enhanced WGM toroid simulations [17]. However, such boundary conditions act as mirrors, effectively replicating the plasmonic particle multiple times, which results in an inaccurate calculation of the coupledsystem Q-factor for a single particle (see Appendix A for more details).

Here we use a three-dimensional (3D) eigenmode simulation model of a whole microtoroid with a major diameter of $10 \mu \mathrm{m}$ to explore the interaction of a single cavity with one or several nanostructures. The model is implemented in COMSOL. Due to simulation constraints in both time and memory, we cannot accurately simulate larger whole toroids in 3D. The schematic of our 3D model and the field distribution of the fundamental TE mode is shown in Fig. 1(a). The polarization of the TE mode is perpendicular to the equatorial plane of the toroid cavity. Nanorods are placed perpendicular to the equatorial plane for maximum 


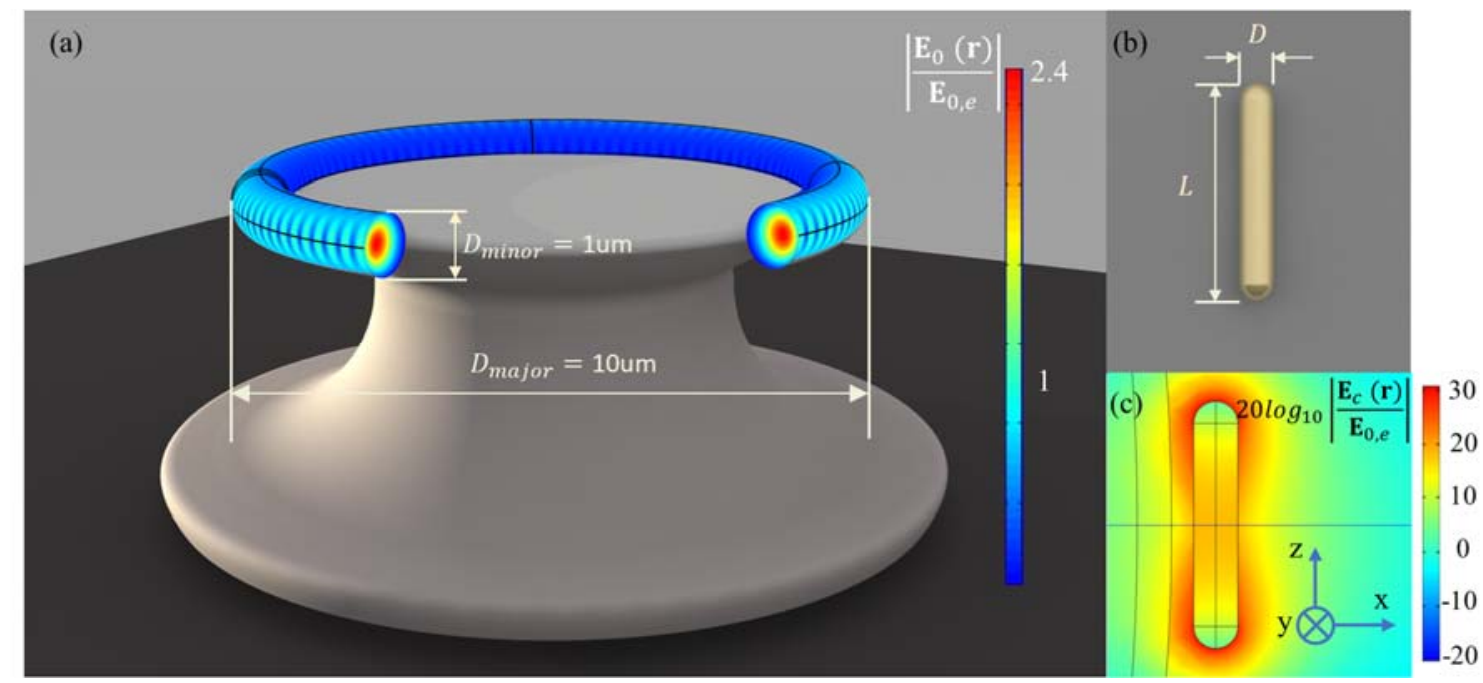

Fig. 1. Schematic of a microtoroid cavity. (a) The E-field is normalized by the amplitude of the maximum field in the evanescent zone of the bare WGM toroid $\left(\mathbf{E}_{o}(\mathbf{r})\right.$ ). (b) A rendering of a gold nanorod placed parallel to the TE polarization of the WGM cavity mode. The resonance frequency of a single rod is tuned by adjusting its aspect ratio, which is defined as the ratio of the length to the width (diameter) of the rod. (c) Field distribution of the excited dipole mode around a nanorod.

coupling and excitation, and their near field enhancement is shown in Fig. 1(c).

We define a figure of merit known as a combined enhancement factor $\left(f_{C}\right)$ to quantify the limit of detection and resolution of our sensing system. With this figure of merit, we can predict that slightly off-resonant coupling between the plasmon and WGM provides better performance than a direct match of the WGM and plasmon resonances. Further, we discuss the improvements that a lateral dark mode supported by a plasmonic trimer structure brings to the system and verify its stability.

Two critical parameters affect the limit of detection of a WGM sensing system. One is the quality factor mentioned above [18], which determines the linewidth of the resonance peak in the output spectrum. Higher quality factors correspond to narrower resonances, whose central resonance frequency can be more precisely determined and tracked $[1-3,19]$. The other parameter is the electric field enhancement factor. The magnitude of the shift in resonance frequency of the WGM upon interaction with an analyte is proportional to this enhancement factor. According to perturbation theory [20], the frequency shift caused by a particle of volume $\Delta V$ with (possibly anisotropic) material parameters $\overleftrightarrow{\Delta \varepsilon}$ (r) and $\overleftrightarrow{\Delta \mu}$ (r) relative to the background is given by [20-22]:

$$
\begin{gathered}
\frac{\Delta \omega}{\omega}=-\frac{\int_{\Delta V}\left[\mathbf{E}_{0}^{\dagger} \varepsilon_{0} \overleftrightarrow{\Delta \varepsilon}(\mathbf{r}) \mathbf{E}+\mathbf{H}_{0}^{\dagger} \mu_{0} \overleftrightarrow{\Delta \mu}(\mathbf{r}) \mathbf{H}\right] d V}{\int_{V}\left[\varepsilon_{0} \varepsilon(\mathbf{r}) \mathbf{E}_{0}^{\dagger} \cdot \mathbf{E}+\mu_{0} \mu(\mathbf{r}) \mathbf{H}_{0}^{\dagger} \cdot \mathbf{H}\right] d V} \\
\approx-\frac{\varepsilon_{b} \mathbf{E}_{0}^{\dagger} \overleftrightarrow{\alpha}(\omega) \mathbf{E}_{0}}{2 \varepsilon_{0} \varepsilon_{r} V_{m}\left|\mathbf{E}_{0}\right|_{\text {max }}^{2}},
\end{gathered}
$$

where $\varepsilon_{0}$ and $\mu_{0}$ are the permittivity and permeability of free space, $\varepsilon$ and $\mu$ are relative permittivities and permeabilities, $\varepsilon_{b}$ is the permittivity of the background media (in this case, vacuum), $\mathbf{E}_{0}$ and $\mathbf{H}_{0}$ are the electric and magnetic fields for an unperturbed $\mathrm{WGM}$, $\dagger$ represents conjugate-transpose, $\mathbf{E}$ and $\mathbf{H}$ are the electric and magnetic fields within the perturbation volume, $\left|\mathbf{E}_{0}\right|_{\max }^{2}$ is the maximum intensity within the unperturbed toroid, $V_{m}=$ $\int_{V} \varepsilon_{r}\left|\mathbf{E}_{0}\right|^{2} d V /\left(\varepsilon_{r}\left|\mathbf{E}_{0}\right|_{\text {max }}^{2}\right)$ is the mode volume of the resonant mode [19], $\varepsilon_{r}$ is the dielectric constant of silica [23], and $\overleftrightarrow{\alpha}(\omega)$ is the polarizability tensor of the particle. In the numerator, we have assumed $\overleftrightarrow{\Delta \mu}=0$, and in the denominator, we have assumed that the magnetic field contributes an equal amount of energy as the electric field and that the perturbation has a negligible impact on the total energy stored in the cavity. The polarizability tensor for a metallic nanorod can be expressed as [24-26]:

$$
\overleftrightarrow{\alpha}(\omega)=\left(\begin{array}{ccc}
\alpha_{\perp} & 0 & 0 \\
0 & \alpha_{\perp} & 0 \\
0 & 0 & \alpha_{\|}
\end{array}\right),
$$

where $\alpha_{\|}$and $\alpha_{\perp}$ correspond to the longitudinal and transverse modes of the nanorod, respectively. The nanorods are small enough that they can be accurately modeled based on the localized surface plasmon resonance of prolate spheroids [25]:

$$
\alpha_{\|, \perp}=\Delta V \varepsilon_{0} \frac{\varepsilon_{m}-\varepsilon_{b}}{G_{\|, \perp} \varepsilon_{m}+\left(1-G_{\|, \perp}\right) \varepsilon_{b}},
$$

where $\Delta V=\frac{4}{3} \pi\left(\frac{d}{2}\right)^{3}+\pi\left(\frac{d}{2}\right)^{2}(L-d)$ is the volume of the cylindrical nanorod, $\varepsilon_{m}$ is the relative permittivity of the metal, $\varepsilon_{b}$ is the dielectric constant of the surrounding environment and the $G_{\|, \perp}$ are geometrical factors that can be respectively written as:

$$
\begin{gathered}
G_{\|}=R_{S} \frac{1-e^{2}}{e^{2}}\left(-1+\frac{1}{2 e} \ln \left(\frac{1+e}{1-e}\right)\right) \\
G_{\perp}=\frac{1-G_{\|}}{2},
\end{gathered}
$$


(a)
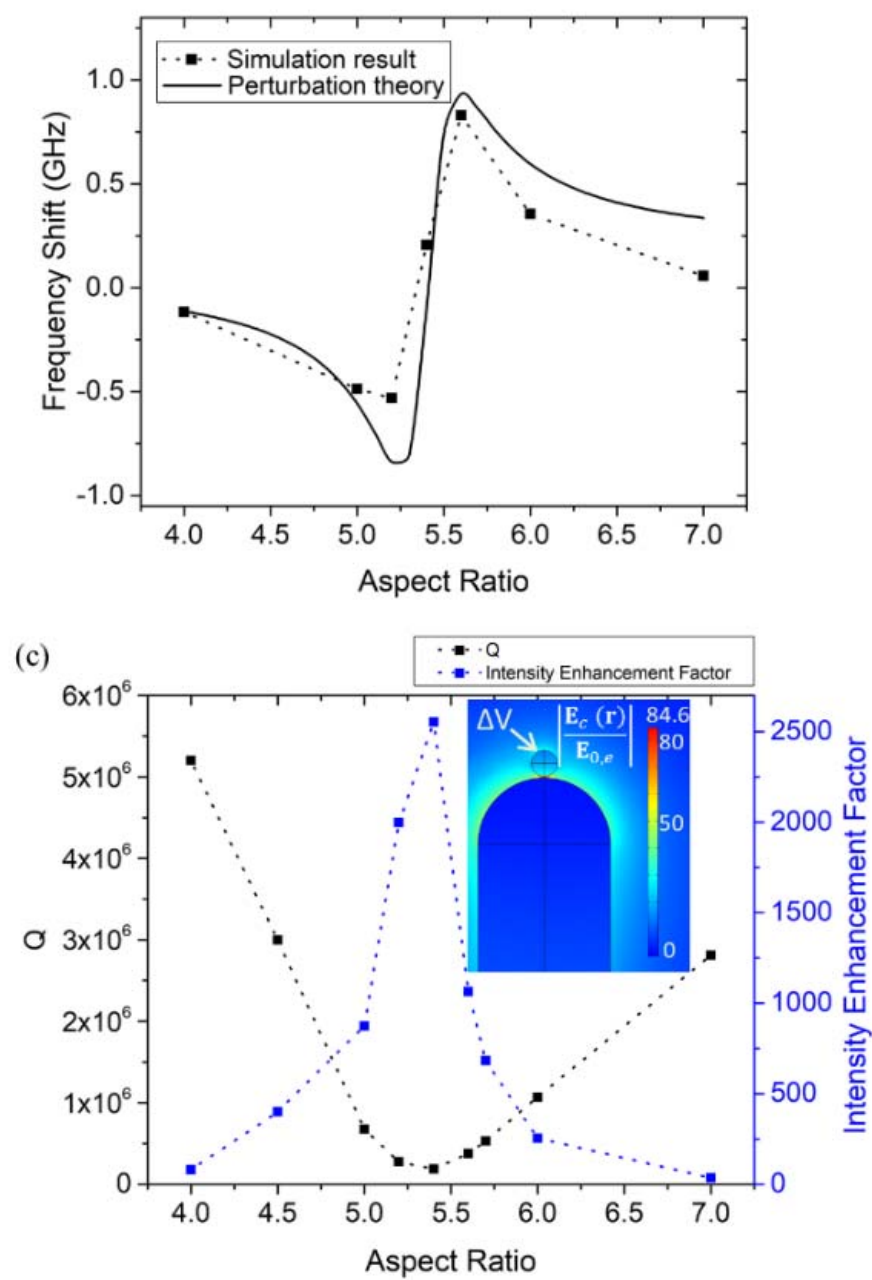

(b)

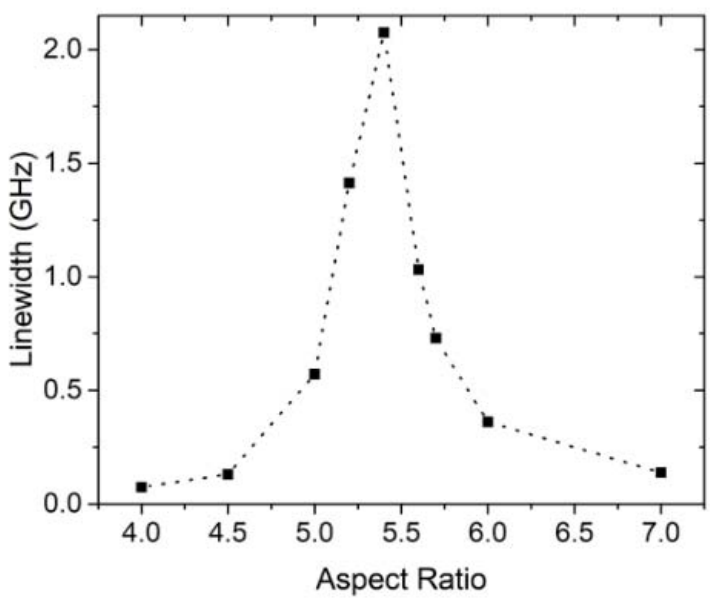

(d)

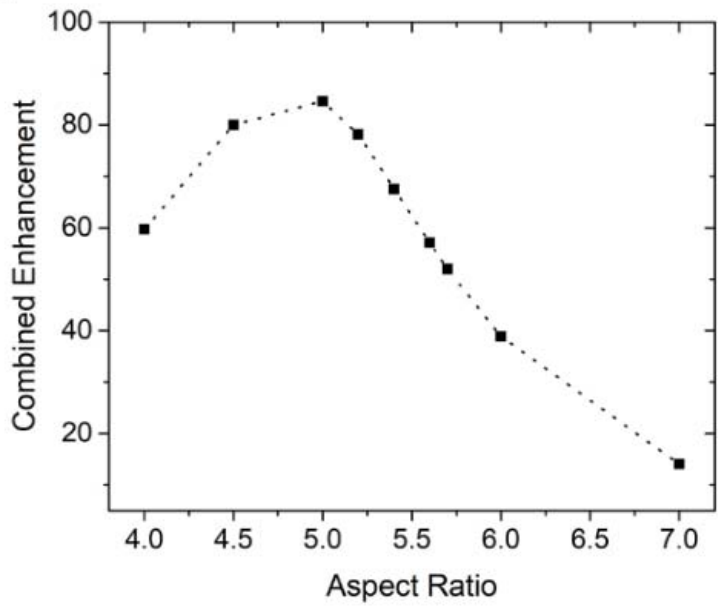

Fig. 2. (a) The blueshift or redshift of the cavity mode around on-resonance coupling. (b) The relationship between the linewidth corresponding to system loss and the resonance of a single rod. (c) The relationship between Q-factor and enhancement as a function of the resonance for a single nanorod. The trend for the enhancement factor is similar to the linewidth change in Fig. 2(b). Extremely strong enhancements are shown for on-resonant coupling. Due to the light-matter interaction, a very strong hot spot is generated between the plasmonic nanorod and the biomolecule. (d) The relationship between the combined enhancement factor $\left(f_{c}\right)$ and the resonance frequency of the rod.

where $e=\sqrt{1-\left(\frac{d}{L}\right)^{2}}$ is the eccentricity of the prolate spheroid and $R_{S}$ is a parameter which describes the particle shape. Here we use $R_{S}=0.88$ for a spherocylinder [27]. In the simulation, we put the rod parallel to the polarization of the fundamental TE mode and study only the influence of the longitudinal plasmon mode and its corresponding $\alpha_{\|}$. It is apparent that $\alpha_{\|}$is greatly influenced by the nanorod aspect ratio. This in turn affects the shift of the unperturbed WGM mode.

\section{COMBINED ENHANCEMENT FACTOR}

Fig. 2(a) illustrates the frequency shift of the cavity mode around on-resonance $(\lambda \approx 774 \mathrm{~nm})$ coupling obtained in the simulation, which is consistent with both experimental data and perturbation theory [28]. The data for the perturbation theory is obtained by numerical calculation of Eqs. (1-4) where only the longitudinal polarizability $\alpha_{\|}$is necessary, and the unperturbed electric fields and mode volumes are determined from a simulation of the bare cavity. In our calculations for different aspect ratios, the nanorod diameter is held fixed while the length is varied, so at large aspect ratios, the assumption that the nanorod size $\ll \lambda$ starts to fail and the theory deviates from the numerical simulation results [29]. At the same time, the linewidth of the coupled system also varies with the resonance frequency of a single rod as shown in Fig. 2(b).

The inset of Fig. 2(c) shows how we calculate the intensity enhancement at the particle, which is done by adding a sphere in the near field of the nanorod to represent 
(a)

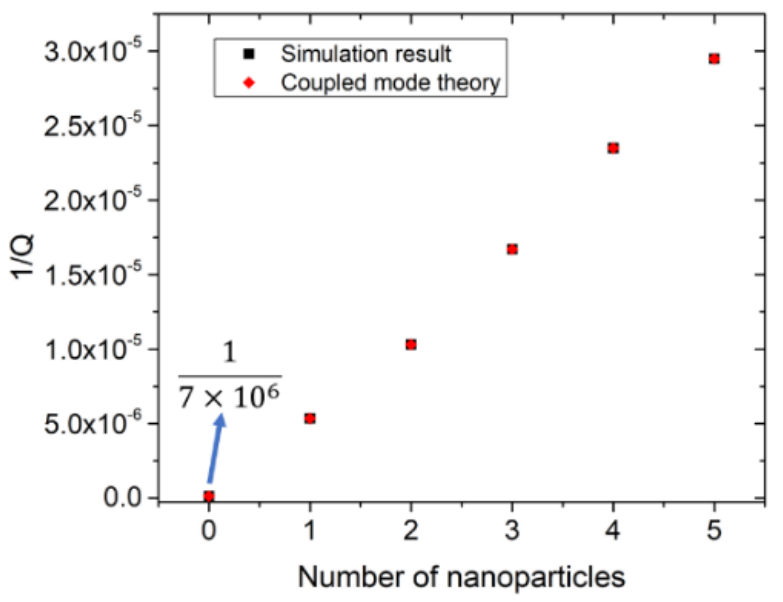

(b)

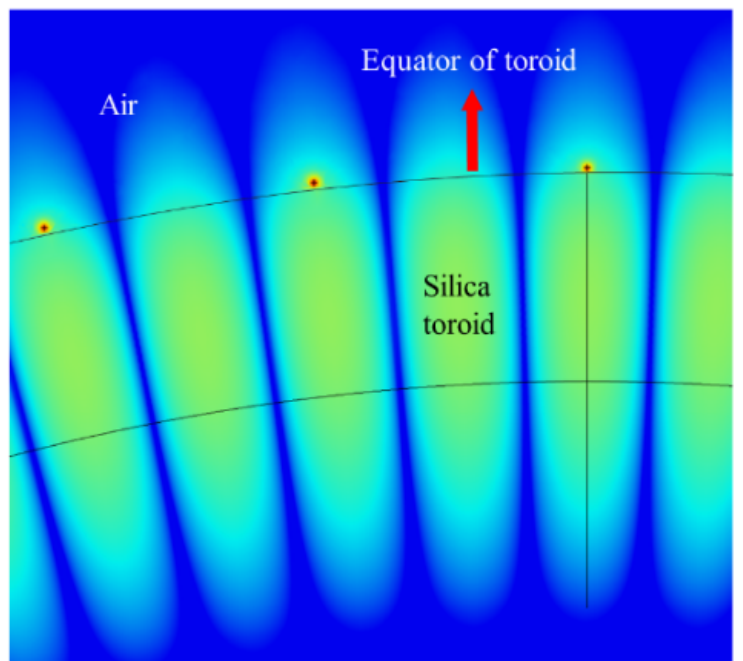

Fig. 3. (a) The Q-factors obtained through both through numerical simulations and coupled mode theory are consistent for the systems involving multiple nanorods and no direct inter-rod coupling. (b) Top view of multiple rods coupled to the cavity mode.

Fig. 3(b) and 2(c) share the same color bar.

an analyte molecule. Here we use the volume integration of the electric field intensity over the volume of an analyte particle as the enhancement factor instead of the maximum E-field intensity at the hot spot of the nanoparticle [10,30]. This is more accurate because the maximum E-field hot spot intensity is sensitive to the mesh of the simulation model and does not take into account the frequency shift caused by the size of the measured particle. The ratio of the integrated field involving a coupled plasmonic particle to that of the evanescent field of the bare toroid can be used to define a field enhancement factor as follows:

$$
f_{E}=\frac{\int_{\Delta V}\left|\mathbf{E}_{c}(\mathbf{r})\right|^{2} d V}{\int_{\Delta V}|\mathbf{E}(\mathbf{r})|^{2} d V},
$$

where $\left|\mathbf{E}_{c}(\mathbf{r})\right|$ is the E-field magnitude of the near field of the coupled system. The enhancement factor corresponding to the electric field intensity directly affects the magnitude of the frequency shift when a particle binds to the cavity. This enhancement factor closely follows the same trend as the linewidth broadening caused by the nanorod (Fig. 2(b)). It is evident that introducing a particle with a lossy material such as gold will significantly decrease the Q-factor of the system even with strong local electric field enhancement, so there is a trade-off between $\mathrm{Q}$ and intensity enhancement factor as shown in Fig. 2(c). At a wavelength of $\lambda \approx 774$ $\mathrm{nm}$, the maximum $2500 \times$ intensity enhancement factor is achieved when the rod aspect ratio equals 5.4. In this case, the peak LSPR wavelength coincides with the particular WGM resonance being simulated. The unperturbed, bare toroid exhibits a Q-factor of $7 \times 10^{6}$, which drops to $1.87 \times 10^{5}$ when coupled to the nanorod with aspect ratio 5.4 .

The lower Q of the coupled system involving resonant nanorods may make it difficult to precisely lock to resonance peaks in experiments involving tracking the wavelength shift of the mode. Therefore, a balance is desired between the Q-factor and the intensity enhancement factor.

From Eqs.1 and 3, it can be shown that frequency shift is directly proportional to the E-field intensity times the volume of the detected particle: $\Delta \omega \propto E^{2} \Delta V$, for scalar $\alpha$. Then, the volume of the smallest detectible particle follows, $\Delta V_{\text {min }} \propto \Delta \omega_{\text {min }} /|E|^{2} \propto\left(Q|E|^{2}\right)^{-1}$, assuming that the smallest experimentally measurable shift, $\Delta \omega_{\min }$, is inversely proportional to $\mathrm{Q}$ factor because high-Q resonances correspond to narrow peaks that can be tracked with greater precision than broad resonances. So, the volume of the smallest detectable particle is inversely proportional to a figure of merit that accounts for both the E-field and Q-factor effects, which we call the combined enhancement factor $\left(f_{C}\right)$ :

$$
f_{C}=f_{Q} f_{E}=\frac{Q_{c}}{Q_{0}} f_{E},
$$

where $Q_{c}$ and $Q_{0}$ represent the Q-factors of the coupled and bare toroid cavity, respectively. Higher $f_{C}$ values correspond to better sensitivity and/or more well-defined resonances. Unlike the peak in $f_{E}$, the highest $f_{C}$ does not occur when the LSPR and WGM resonances coincide. Instead, as shown in Fig. 2(d), the position of the peak is shifted significantly to a smaller aspect ratio than that of resonant coupling. We can state that the improvement to the system from a single rod with an aspect ratio of 5.0 is ideal, which corresponds to a $874 \times$ field enhancement factor and a Q-factor of $6.8 \times 10^{5}$. Using $f_{C}$ as a figure of merit is helpful in selecting the best plasmonic structure for experimental use.

\section{INDIVIDUAL NANORODS COUPLED SYSTEM}

For multiple gold nanoantennas, we investigate the intrinsic properties of the coupled system. The single gold nanorod can be approximated as a nanocavity. According to coupled mode theory [21,31-33], the equations of several coupled modes can be written as: 
(a)

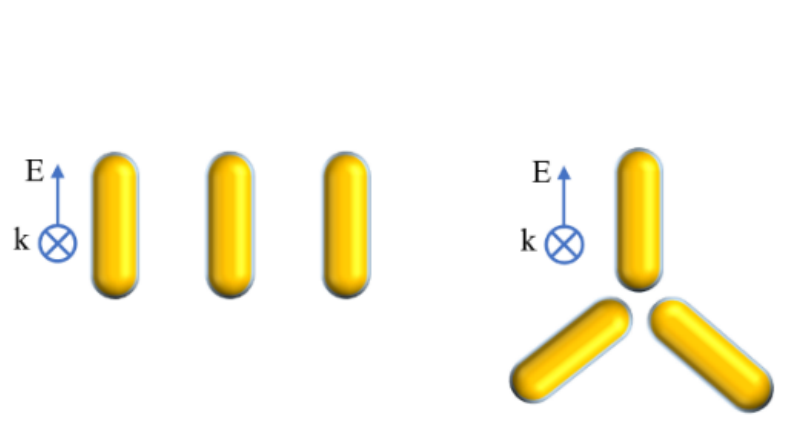

(c)

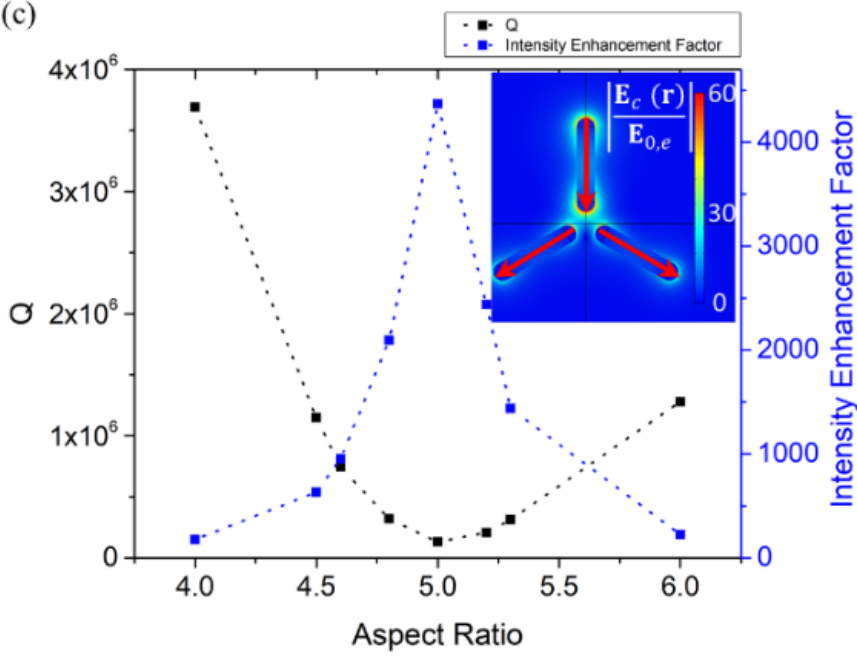

(b)

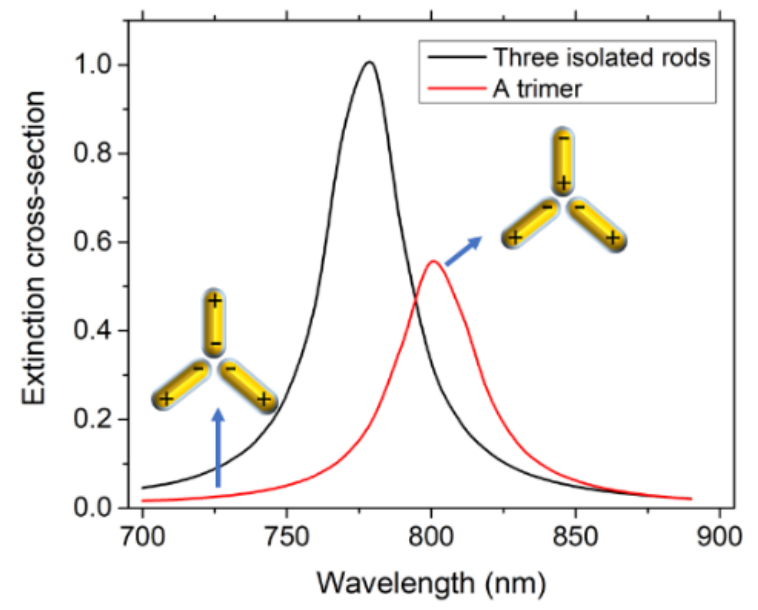

(d)

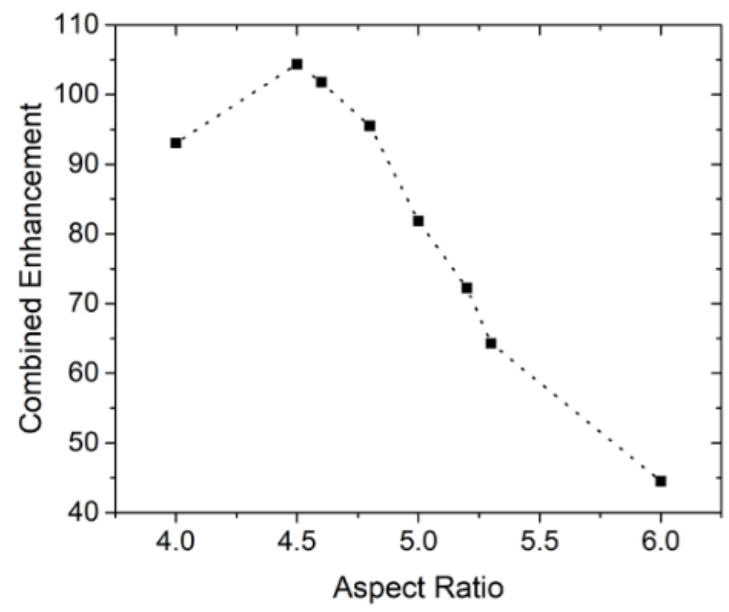

Fig. 4. (a) Plane wave excitation for three isolated rods and a gold trimer. (b) Spectral comparison of the total extinction cross section of the lateral dark mode versus the three isolated rods from Fig. 4(a). The lateral dark mode is excited at the peak wavelength of the red curve. The illustrations show the charge distribution of the breathing and lateral dark mode at different wavelengths. Because the coupling between the breathing dark mode and free-space radiation is so small, no peak is visible at its resonance around $725 \mathrm{~nm}$. (c) Scan of two parameters of the trimer (lateral dark mode) coupling system by tuning the aspect ratio of the individual rods. The red arrows show the current density direction obtained in COMSOL. The inset shows the field distribution of excited lateral dark mode. The characteristic dark spot between the ends of the bottom two rods is clearly visible. (d) Plot of the $f_{C}$ of the trimer. The lateral dark mode exhibits a higher combined enhancement value than that obtained from the coupling a single rod to the cavity alone.

$$
\begin{gathered}
\frac{d b_{\mathrm{WGM}}}{d t}=\left(i \Omega_{W G M}-\Gamma_{W G M}\right) b_{\mathrm{WGM}}+i \kappa b_{1}+i \kappa b_{2}+\cdots \\
\quad+i \kappa b_{n} \\
\frac{d b_{1}}{d t}=\left(i \Omega_{1}-\Gamma_{1}\right) b_{1}+i \kappa b_{\mathrm{WGM}} \\
\vdots \\
\frac{d b_{n}}{d t}=\left(i \Omega_{n}-\Gamma_{n}\right) b_{n}+i \kappa b_{\mathrm{WGM}}
\end{gathered}
$$

where $b_{\mathrm{WGM}}$ and $b_{n}$ represent the mode amplitude of the WGM mode and LSPR mode of the nth nanorod, and $\Omega_{W G M}$ and $\Omega_{n}$ correspond to their resonance frequencies and $\Gamma_{W G M}$ and $\Gamma_{n}$ represent their corresponding losses, respectively, which can be calculated from COMSOL using the eigenfrequency solver. The coupling coefficient is defined as a complex number $\kappa$ which takes into account the frequency shift of the mode and the degradation of the Q. Because the rods in the array are spaced relatively far apart, we only consider the coupling of each LSPR mode with the cavity and no direct interparticle coupling. Therefore, the above equations for the coupled system can be written as a matrix equation $\dot{\mathrm{M}}=H \mathrm{M}$, where $\mathbf{M}=\left[b_{W G M}, b_{1}, \ldots b_{n}\right]^{T}$, and

$$
H=\left[\begin{array}{cccc}
\Omega_{W G M}+i \Gamma_{W G M} & \kappa & \cdots & \kappa \\
\kappa & \Omega_{1}+i \Gamma_{1} & 0 & 0 \\
\vdots & 0 & \ddots & \vdots \\
\kappa & 0 & \cdots & \Omega_{n}+i \Gamma_{n}
\end{array}\right]
$$


(a)

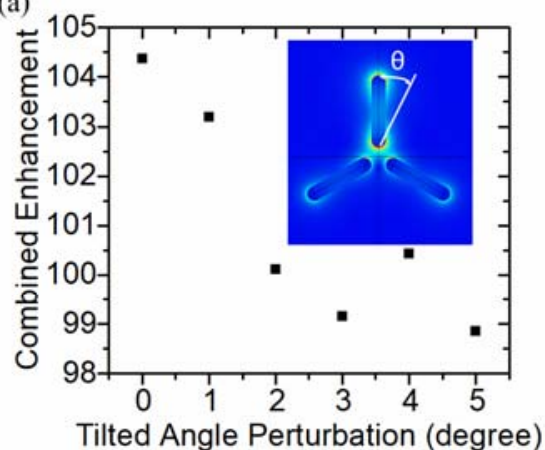

(b)

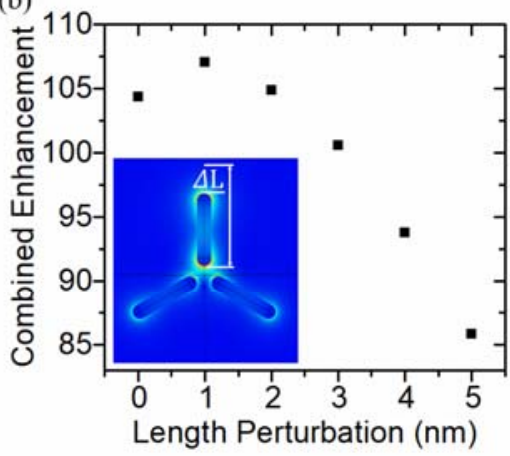

(c)

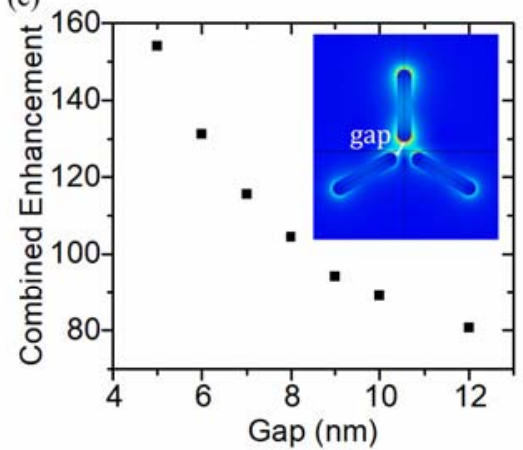

(d)

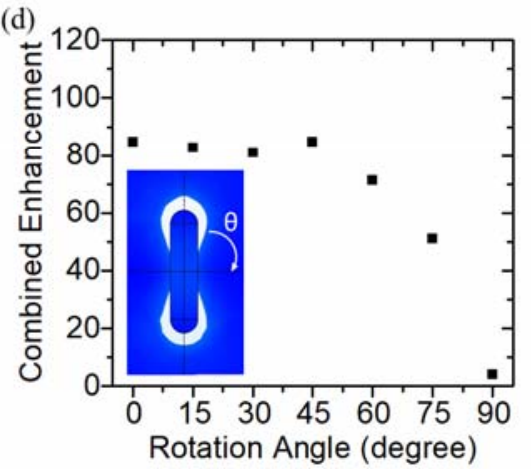

(e)

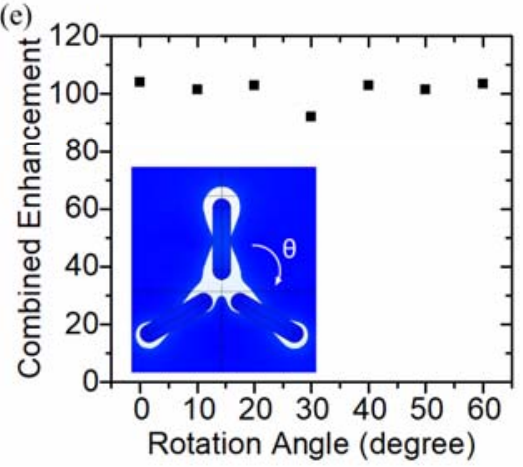

Fig. 5. Influence of different perturbations on the trimer's combined enhancement factor (a) and (b) Small changes in angle and length can maintain the $f_{c}$. (c) To ensure that the gap space is large enough for particles to bind, we study the effect of the spacing on the system when the spacing is greater than $5 \mathrm{~nm}$. The illustrations for the trimer field distribution use the same color bar as in Fig. 3. (d) The $f_{c}$ of a single rod system decreases with increasing rotation angle. When the rod is rotated 90 degrees, that is, perpendicular to the polarization of the TE mode, the overall system improvement $f_{C}$ is only $4 \times$. The white area in the inset shows the where the

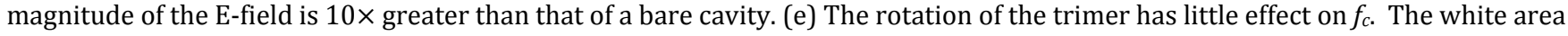
in the inset shows the where the magnitude of the E-field is $10 \times$ greater than that of a bare cavity. The enhancement area provided by the trimer (e) is slightly more than twice that of a single rod (d).

The intrinsic $\mathrm{Q}$ factor of the coupled system can be calculated from the eigenvalues of $H$, corresponding to the steady-state of the system. Through the simulation data, the coupling coefficient $\kappa\left(\sim 1.43 \times 10^{11} \mathrm{~Hz}\right)$ can be obtained from a $2 \times 2$ matrix generated by a system in which a cavity is coupled to a single rod (see Appendix B). Further, the $\mathrm{Q}$ factor of a $(n+1) \times(n+1)$ matrix corresponding to a multiple (n) rod coupled system can be calculated numerically. We compare these values with the $\mathrm{Q}$ factors obtained from COMSOL simulation of the multi-rod system (Fig. 3(a)). The coupled mode theory can then be used to predict the interaction between the WGM and additional particles without having to run new COMSOL simulations.

Fig. 3(a) also shows the full simulation results of coupling several rods to the cavity, where each rod is placed at an antinode of the standing wave WGM (Fig. 3(b)). Here, the nanorods are sufficiently far apart such that direct coupling between neighboring nanorods is negligible compared to nanorod-WGM coupling, as verified in Fig. 3(a) by the agreement between the numerical simulation results and coupled mode theory (eigenvalues of $H$, Eq. 9).

\section{TRIMER-INDUCED LATERAL DARK MODE}

The dipole modes induced in isolated antennas lead to large energy radiation which adversely limit the Q-factors of the coupled systems. Dark modes, an example of which is the antibonding interaction of dipolar resonances, have a longer lifetime and lower loss compared to bright dipolar modes, but their excitation is not easily feasible from the farfield $[12,13,16]$. Due to the nearly zero net dipole moment of dark plasmons, it is almost impossible for their out-ofphase state to interact with light in free space.

Here we designed a plasmonic trimer consisting of three isolated rods arranged in a symmetric triangle [34,35] (Fig. 4(a)) such that the microtoroid WGM excites one of the nanorods in the trimer, which in turn excites a dark mode resonance (lateral dark mode) of the other two nanorods [12,15,36-38]. Coupling of the dark mode and WGM standing wave can significantly reduce degradation of the Q-factor. The superposition of rod near-fields at the center of the trimer can also bring greater intensity enhancement factors. Fig. 4(b) shows the spectrum of the lateral dark mode of a trimer and three individual rods in free space. When the ends of three rods exhibit the same charge distribution, we call it a breathing dark mode. Only when two antennas of the trimer generate an antibonding mode (Fig. 4(b)) do we refer to this as a lateral dark mode. Although the trimer spectrum exhibits a peak near $800 \mathrm{~nm}$ upon far-field or WGM excitation, we still call its mode a 
lateral dark mode because the laterally-oriented lower two rods of the trimer are excited in an anti-bonding, or dark, mode. The apparent resonance peak in Fig. 4(b) is due to the excitation of the vertical rod, which remains bright. Because of this simultaneous combination of bright and dark modes, it is possible to excite a plasmon resonance with minimal scattering and absorption losses. Although the breathing dark mode has much lower loss and its corresponding resonance peak is almost covered by the tail of the lateral dark mode, its coupling to the WGM is very weak. Therefore, this paper mainly studies the lateral dark mode instead of the breathing dark mode.

Due to the plasmonic coupling between closely-placed nanorods, the resonance position of the lateral dark mode shifts with respect to the spacing and position of the rods. In Fig. 4(b), we compare the extinction cross sections when three rods with the same aspect ratio of 5.4 are placed separately or placed as a trimer with an $8 \mathrm{~nm}$ central gap. The extinction cross sections are normalized by that of the three isolated rods. The maximum extinction of the trimer is about half of that obtained from three individual rods. At many wavelengths, including $774 \mathrm{~nm}$, corresponding to the WGM resonance, the loss of from the trimer is even lower than that of a single rod. Such a low-dissipation mode improves the WGM sensing system.

We also adjust the aspect ratio of the three rods in the trimer to optimize the improvement of the trimer coupling system. Fig. 4(c) shows how the Q-factor and intensity enhancement vary with the rod aspect ratio. The biggest intensity enhancement factor here corresponds to the case where the spherical analyte particle is placed in the center of the trimer. At an aspect ratio of 5, the maximum field enhancement factor $(\sim 4300 \times)$ is much larger than that of a single rod $(\sim 2500 \times)$, mentioned above. At the same time, the Q-factor of $1.31 \times 10^{5}$ is similar to that of the single rod case $\left(\sim 1.87 \times 10^{5}\right)$ at on-resonance coupling and is twice as large as the $\mathrm{Q}$ for the three rod case $\left(\sim 5.98 \times 10^{4}\right)$ presented in Fig. 3(a). Even the relatively dark region just below the center of the trimer in the inset of Fig. 4(c) can bring dozens of times of intensity enhancement, which also demonstrates that the trimer structure is beneficial to increase capture area for biomolecule detection. The maximum $f_{C}$ of the system is $\sim 105$, which is achieved using the trimer with rod aspect ratio 4.5 . This $f_{C}$ is also significantly larger than that of a single rod $(\sim 84)$.

To test the robustness of our system, we studied the effect of tilted angle perturbation, length perturbation, and inter-rod spacing on $f_{C}$. Here we chose the trimer with a rod aspect ratio of 4.5 mentioned above as a reference. As shown in the insets in Fig. 5, we observe the difference of $f_{C}$ in the system by introducing different geometric perturbations. Considering the need to bind biomolecules at the center of the trimer in experiments, the spacing between the rods should not be too small. Although different perturbations will impact the Q-factor or enhancement factor of the system, the overall $f_{C}$ changes little from its initial value 105. The spacing in Fig. 5(c) has little effect on the loss caused by the structure, so the corresponding $\mathrm{Q}$ value is almost unchanged, and the observed variation is primarily due to E-field enhancement.

Rotations of the entire structure are evaluated for single nanorods and trimer structures in Figs. 5(d) and 5(e). The gold nanorods in current plasmonic-enhanced WGM systems are randomly attached on the surface of the cavity and their orientation is challenging to control. Here we use a rod with an aspect ratio of 5 (Fig. 2) to study the influence of the rotation angle on $f_{C}$. When the rotation angle is greater than 45 degrees, the $f_{C}$ decreases as the rotation angle increases (Fig. 5(d)) due to mismatch with the polarization direction of the WGM. The system has only $4 \times$ gain when rotated 90-degrees. In comparison, the trimer is robust to orientation changes. Due to its rotational symmetry, we only rotate the whole trimer from 0 to 60 degrees (Fig. 5(e)). The 0 -degree point corresponds to the idealized trimer composed of three rods with an aspect ratio of 4.5. The weakest $f_{C}$ at 30 -degree angle is $92 \times$, which is only $11.5 \%$ lower than the maximum enhancement $104 \times$. The trimer system is much less affected by the rotation angle than the single rod system. The inconsistent signal amplification caused by the rotation of a single rod can add uncertainty to particle detection experiments.

\section{CONCLUSIONS}

In this work, we present a lateral dark mode of a symmetric trimer structure generated using whispering gallery mode excitation and define a combined enhancement factor to analyze the improvements it brings to the system. The trimer exhibits a $\sim 105 \times$ combined enhancement factor vs $84 \times$ for a single rod. We also demonstrate the stability and robustness of the trimer structure. We verify the correctness of our simulation from the frequency shift and linewidth changes caused by detuning and the effect on Q-factor. Compared to the existing single-rod plasmonic hybrid WGM system, the trimer system exhibits higher enhancement, stability, and greater contact area for particle detection. In future work, we plan to use high-precision optical tweezers $[39,40]$ to position trimer microresonator systems for biological sensing experiments.

\section{APPENDIX A: THE DETAILS OF THE FULL 3D TOROID SIMULATION AND ITS COMPARISON WITH THE WEDGE MODEL}

The data and profiles are obtained in COMSOL using finite element analysis. The material of the cavity is silica with a refractive index of $1.45+10^{-8} i$ and the background is air [18] with a refractive index of 1 . We add the imaginary component of the refractive index of the cavity to lower the Q-factor to a value similar to what we would see in experiment. The material parameters of gold used here are those given by Johnson and Christy [41]. The diameter of each gold rod is $6 \mathrm{~nm}$. The simulated biomolecule used to calculate the enhancement factor is a sphere with a refractive index of 1.5 and a radius of $1 \mathrm{~nm}$. We constructed 
(a)

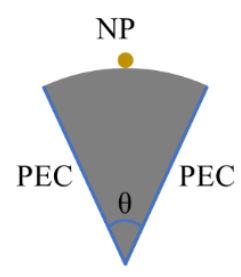

(d)

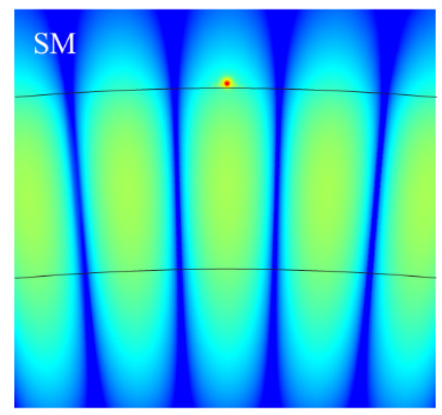

(b)

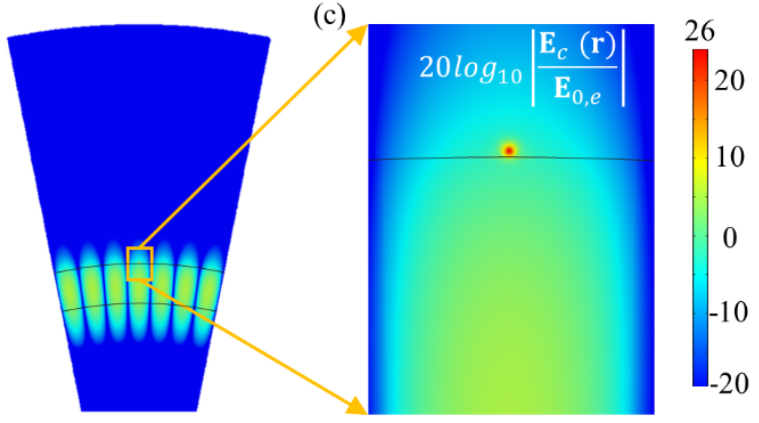

(e)

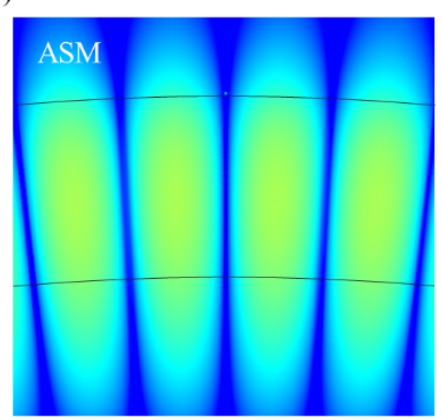

Fig. A1. (a) Schematic of the wedge model. (b) Electric field distribution of a particle coupled to a toroid cavity (top view). The particle is placed at the antinode. (c) A zoom-in of near field enhancement. (d) and (e) show the symmetric and asymmetric modes of the coupled system obtained by the whole 3D model using eigenfrequency solver, respectively. The corresponding detuning frequency between the SM and ASM modes is $200 \mathrm{MHz}$.

the mesh of the cavity by using a uniform sweeping method with a maximum size of one-ninth of a wavelength. The Qfactor and field distribution of the unperturbed cavity surrounded by perfectly matched layer are consistent with the analytical solution and the two-dimensional axisymmetric model [42-45]. The Q is calculated from the real and imaginary parts of the eigenfrequency obtained by COMSOL. In the matrix given in Eq. 5, the parameters $\Omega_{W G M}\left(3.8724 \times 10^{14} \mathrm{~Hz}\right)$ and $\Gamma_{W G M}\left(1.03 \times 10^{9} \mathrm{~Hz}\right)$ are determined from simulations of a bare toroid and the interaction of a single rod with a cavity using the eigenfrequency solver in COMSOL. $\Omega_{n}(3.8724 \times$ $\left.10^{14} \mathrm{~Hz}\right)$ and $\Gamma_{n}\left(1.479 \times 10^{12} \mathrm{~Hz}\right)$ are obtained from the spectrum of the nanoparticle in free space, and all rods are identical. The server used for the simulation is configured as a 56-core $2.3 \mathrm{GHz}$ processor and $384 \mathrm{~GB}$ memory.

The wedge simulation model is currently widely used in the calculation and prediction of relevant experimental data for microresonators [17]. The model uses perfect electric conductor (PEC) boundary conditions which can be considered as mirrors. Such boundary conditions apply only to bare cavities, but when the cavity is coupled to a nanoparticle (NP) or structure, the PEC boundary conditions replicate the nanoparticles multiple times, such that the interaction between one nanoparticle and the WGM cannot be accurately predicted. This part compares the results from a wedge model with those of the full 3D model mentioned in the main body of the paper.
Here we study the effect of the wedge angle on the Q value. The $Q$ value decreases with the increasing number of coupled NPs. To compare the wedge model with a whole $3 \mathrm{D}$ model, we construct both models for a toroid with a major radius of 5 microns. Fig. A1(a) shows the schematic of the wedge model. The wedge angle $\theta$ is inversely proportional to the number of azimuthal modes $m$, and can be written as:

$$
\theta=N \frac{\pi}{m},
$$

where $N$ is an integer corresponding to the number of antinodes of the standing wave in Fig. A1(b). $m$ satisfies the resonance condition $m \lambda=2 \pi n_{\text {eff }} R$, where $n_{\text {eff }}$ represents the effective refractive index of the mode. The $N$ in the wedge structure is taken as $1,3,5,7$ and compared with the whole toroid model which is used in the main text. The corresponding field distribution for the $N=7$ case is shown in Figs. A1(b) and (c).

Table A1. Q-factor of the coupled system for different wedge angles. The last column is the result of the whole toroid model coupled to a single rod of the same size.

\begin{tabular}{|l|l|l|l|l|c|}
\hline $\mathrm{N}$ & 1 & 3 & 5 & 7 & $\begin{array}{l}\text { Whole } \\
\text { toroid }\end{array}$ \\
\hline $\begin{array}{l}\text { Q- } \\
\text { factor }\end{array}$ & $\begin{array}{l}8.10 \\
\times 10^{3}\end{array}$ & $\begin{array}{l}2.41 \\
\times 10^{4}\end{array}$ & $\begin{array}{l}4.04 \\
\times 10^{4}\end{array}$ & $\begin{array}{l}5.62 \\
\times 10^{4}\end{array}$ & $\begin{array}{l}1.87 \\
\times 10^{5}\end{array}$ \\
\hline
\end{tabular}

The Q of the wedge structure is significantly lower than that of the whole toroid and one particle. This may be because the larger the wedge angle, the lower the number of 
coupled particles, so the higher the Q. But we don't know the interaction of a single rod and a cavity, nor can we calculate the influence of boundary conditions on the electric field and its influence on the coupled system.

Furthermore, when a nano-scatterer is coupled to the WGM cavity, the coupling of the scattered light to the clockwise mode and counter-clockwise mode of the cavity will generate both a symmetric mode (SM) and an asymmetric (ASM) mode [46]. Symmetric and asymmetric modes correspond to the antinode or node of the standing wave where the particle is located, respectively. Since the PEC boundary condition sets the electric field to zero at the boundary, the position of the WGM standing wave at a specific wavelength is fixed. Accordingly, the introduced nanoparticle does not interact with both the SM and ASM simultaneously. The 3D whole toroid model, however, can generate two modes as shown in Figs. A1(d) and (e). This is another advantage of the whole toroid model. Therefore, the whole toroid model is used here rather than the wedge model.

\section{APPENDIX B: COUPLING COEFFICIENT CALCULATION}

$\Omega_{W G M}+i \Gamma_{W G M}$ and $\Omega_{1}+i \Gamma_{1}$ can be obtained from the eigenfrequency in the bare toroid simulation and the isolated nanorod, respectively. If we assume that the complex eigenfrequency of the coupled system is $\Omega_{\text {coupled }}+$ $i \Gamma_{\text {coupled }}$ which can be solved from the simulation of the bare toroid coupled to a single nanorod, then from the analytical eigenvalue of the $2 \times 2$ matrix, we can calculate $\kappa$ using:

$$
\begin{gathered}
\kappa=\left[( \Omega _ { W G M } + i \Gamma _ { W G M } - \Omega _ { \text { coupled } } - i \Gamma _ { \text { coupled } } ) \left(\Omega_{1}+i \Gamma_{1}\right.\right. \\
\left.\left.-\Omega_{\text {coupled }}-i \Gamma_{\text {coupled }}\right)\right]^{\frac{1}{2}}
\end{gathered}
$$

Funding. Defense Threat Reduction Agency (DTRA) (HDTRA11810044); The University of Arizona; DeMund Foundation Graduate Student Endowed Scholarship in Optical and Medical Sciences, Friends of Tucson Optics (FOTO) Scholarship.

\section{REFERENCES}

1. J. Su, "Label-Free Single Exosome Detection Using FrequencyLocked Microtoroid Optical Resonators," ACS Photonics 2, 1241-1245 (2015).

2. J. Su, "Label-Free Biological and Chemical Sensing Using Whispering Gallery Mode Optical Resonators: Past, Present, and Future," Sensors 17, 540 (2017).

3. J. Su, A. F. Goldberg, and B. M. Stoltz, "Label-free detection of single nanoparticles and biological molecules using microtoroid optical resonators," Light: Science \& Applications 5, e16001 (2016).

4. J. D. Swaim, J. Knittel, and W. P. Bowen, "Detection limits in whispering gallery biosensors with plasmonic enhancement," Appl. Phys. Lett. 99, 243109 (2011).

5. F. Vollmer and L. Yang, "Review Label-free detection with highQ microcavities: a review of biosensing mechanisms for integrated devices," Nanophotonics 1, 267-291 (2012).
6. Y. Zhi, X.-C. Yu, Q. Gong, L. Yang, and Y.-F. Xiao, "Single Nanoparticle Detection Using Optical Microcavities," Advanced Materials 29, 1604920 (2017).

7. D. K. Armani, T. J. Kippenberg, S. M. Spillane, and K. J. Vahala, "Ultra-high-Q toroid microcavity on a chip," Nature 421, 925-928 (2003).

8. M. R. Foreman, J. D. Swaim, and F. Vollmer, "Whispering gallery mode sensors," Advances in Optics and Photonics 7, 168 (2015).

9. F. Vollmer, S. Arnold, D. Braun, I. Teraoka, and A. Libchaber, "Multiplexed DNA Quantification by Spectroscopic Shift of Two Microsphere Cavities," Biophys J 85, 1974-1979 (2003).

10. M. D. Baaske, M. R. Foreman, and F. Vollmer, "Single-molecule nucleic acid interactions monitored on a label-free microcavity biosensor platform," Nature Nanotechnology 9, 933-939 (2014).

11. S. I. Shopova, R. Rajmangal, S. Holler, and S. Arnold, "Plasmonic enhancement of a whispering-gallery-mode biosensor for single nanoparticle detection," Appl. Phys. Lett. 98, 243104 (2011).

12. Y. Gao, N. Zhou, Z. Shi, X. Guo, and L. Tong, "Dark dimer mode excitation and strong coupling with a nanorod dipole," Photon. Res., PRJ 6, 887-892 (2018).

13. T.-S. Deng, J. Parker, Y. Yifat, N. Shepherd, and N. F. Scherer, "Dark Plasmon Modes in Symmetric Gold Nanoparticle Dimers Illuminated by Focused Cylindrical Vector Beams," J. Phys. Chem. C 122, 27662-27672 (2018).

14. D. E. Gómez, Z. Q. Teo, M. Altissimo, T. J. Davis, S. Earl, and A. Roberts, "The Dark Side of Plasmonics," Nano Letters 13, 3722$3728(2013)$.

15. D. E. Gómez, K. C. Vernon, and T. J. Davis, "Symmetry effects on the optical coupling between plasmonic nanoparticles with applications to hierarchical structures," Physical Review B 81, (2010).

16. J.-S. Huang, J. Kern, P. Geisler, P. Weinmann, M. Kamp, A. Forchel, P. Biagioni, and B. Hecht, "Mode Imaging and Selection in Strongly Coupled Nanoantennas," Nano Letters 10, 2105-2110 (2010).

17. A. Kaplan, M. Tomes, T. Carmon, M. Kozlov, O. Cohen, G. Bartal, and H. G. L. Schwefel, "Finite element simulation of a perturbed axial-symmetric whispering-gallery mode and its use for intensity enhancement with a nanoparticle coupled to a microtoroid," Opt. Express, OE 21, 14169-14180 (2013).

18. M. L. Gorodetsky, A. A. Savchenkov, and V. S. Ilchenko, "Ultimate Q of optical microsphere resonators," Opt. Lett., OL 21, 453-455 (1996).

19. T. J. A. Kippenberg, "Nonlinear Optics in Ultra-high Q Whispering-Gallery Optical Microcavities," PhD, California Institute of Technology (2004).

20. R. A. Waldron, "Perturbation theory of resonant cavities," Proceedings of the IEE - Part C: Monographs 107, 272-274 (1960).

21. H. A. Haus, Waves and Fields in Optoelectronics (Prentice-Hall , 1984).

22. S. Arnold, M. Khoshsima, I. Teraoka, S. Holler, and F. Vollmer, "Shift of whispering-gallery modes in microspheres by protein adsorption," Optics Letters 28, 272 (2003).

23. L. Novotny and B. Hecht, Principles of Nano-Optics (Cambridge University Press, 2012).

24. H. Chen, L. Shao, Q. Li, and J. Wang, "Gold nanorods and their plasmonic properties," Chemical Society Reviews 42, 2679-2724 (2013).

25. D. P. Sprünken, H. Omi, K. Furukawa, H. Nakashima, I Sychugov, Y. Kobayashi, and K. Torimitsu, "Influence of the Local Environment on Determining Aspect-Ratio Distributions of Gold Nanorods in Solution Using Gans Theory," The Journal of Physical Chemistry C 111, 14299-14306 (2007).

26. C. F. Bohren and D. R. Huffman, Absorption and Scattering of Light by Small Particles (John Wiley \& Sons, 2008).

27. S. W. Prescott and P. Mulvaney, "Gold nanorod extinction spectra," Journal of Applied Physics 99, 123504 (2006).

28. F. Ruesink, H. M. Doeleman, E. Verhagen, and A. F. Koenderink, "Controlling nanoantenna polarizability through backaction via a single cavity mode," Physical Review Letters 120, (2018). 
29. L. Novotny, "Effective Wavelength Scaling for Optical Antennas," Physical Review Letters 98, (2007).

30. V. R. Dantham, S. Holler, C. Barbre, D. Keng, V. Kolchenko, and S. Arnold, "Label-Free Detection of Single Protein Using a Nanoplasmonic-Photonic Hybrid Microcavity," Nano Letters 13, 3347-3351 (2013).

31. B. E. Little, S. T. Chu, H. A. Haus, J. Foresi, and J.- Laine, "Microring resonator channel dropping filters," Journal of Lightwave Technology 15, 998-1005 (1997).

32. Wonjoo Suh, Zheng Wang, and Shanhui Fan, "Temporal coupledmode theory and the presence of non-orthogonal modes in lossless multimode cavities," IEEE Journal of Quantum Electronics 40, 1511-1518 (2004).

33. A. Yariv, "Universal relations for coupling of optical power between microresonators and dielectric waveguides," Electronics Letters 36, 321 (2000).

34. J. Alegret, T. Rindzevicius, T. Pakizeh, Y. Alaverdyan, L. Gunnarsson, and M. Käll, "Plasmonic Properties of Silver Trimers with Trigonal Symmetry Fabricated by Electron-Beam Lithography," J. Phys. Chem. C 112, 14313-14317 (2008).

35. S. L. Teo, V. K. Lin, R. Marty, N. Large, E. A. Llado, A. Arbouet, C. Girard, J. Aizpurua, S. Tripathy, and A. Mlayah, "Gold nanoring trimers: a versatile structure for infrared sensing," Optics Express 18, 22271 (2010).

36. T. J. Davis, D. E. Gómez, and K. C. Vernon, "Simple Model for the Hybridization of Surface Plasmon Resonances in Metallic Nanoparticles," Nano Lett. 10, 2618-2625 (2010).

37. Funston Alison M., Davis Timothy J., Novo Carolina, and Mulvaney Paul, "Coupling modes of gold trimer superstructures," Philosophical Transactions of the Royal Society A: Mathematical, Physical and Engineering Sciences 369, 3472-3482 (2011).
38. T. J. Davis, K. C. Vernon, and D. E. Gómez, "Designing plasmonic systems using optical coupling between nanoparticles," Physical Review B 79, (2009).

39. A. Ashkin, J. M. Dziedzic, J. E. Bjorkholm, and S. Chu, "Observation of a single-beam gradient force optical trap for dielectric particles," Opt. Lett., OL 11, 288-290 (1986).

40. J. E. Melzer and E. McLeod, "Fundamental Limits of Optical Tweezer Nanoparticle Manipulation Speeds," ACS Nano 12, 2440-2447 (2018).

41. P. B. Johnson and R. W. Christy, "Optical Constants of the Noble Metals," Phys. Rev. B 6, 4370-4379 (1972).

42. M. Oxborrow, "Traceable 2-D Finite-Element Simulation of the Whispering-Gallery Modes of Axisymmetric Electromagnetic Resonators," IEEE Transactions on Microwave Theory and Techniques 55, 1209-1218 (2007).

43. J.-P. Berenger, "A perfectly matched layer for the absorption of electromagnetic waves," Journal of Computational Physics 114, 185-200 (1994).

44. B. Min, L. Yang, and K. Vahala, "Perturbative analytic theory of an ultrahigh- Q toroidal microcavity," Physical Review A 76, 013823 (2007).

45. M. Oxborrow, "Configuration of COMSOL Multiphysics for simulating axisymmetric dielectric resonators: explicit weak-form expressions axisymmetric electromagnetic resonators,"

http://resource.npl.co.uk/docs/science_technology/time_frequency /mwave_standards/axisymmetric/comsol_config.pdf.

46. J. Zhu, S. K. Ozdemir, Y.-F. Xiao, L. Li, L. He, D.-R. Chen, and L. Yang, "On-chip single nanoparticle detection and sizing by mode splitting in an ultrahigh- $Q$ microresonator," Nature Photonics 4, 46-49 (2010). 